\title{
A Comparison of Quantitative Distribution of Nerve Fiber Bundles between Dermatofibroma with and without Pain by Demonstration of Protein Gene Product 9.5 Expression
}

\author{
Kyung-Nam Bae ${ }^{1,2}$, Hyun-Joo Lee ${ }^{1,2}$, Sung-Min Park ${ }^{1,2}$, So Jeong Lee ${ }^{2,3}$, Moon-Bum Kim ${ }^{1,2}$, \\ Min-Young Yang ${ }^{1,2}$ \\ ${ }^{1}$ Department of Dermatology, Pusan National University School of Medicine, ${ }^{2}$ Biomedical Research Institute, Pusan National University \\ Hospital, ${ }^{3}$ Department of Pathology, Pusan National University Hospital, Pusan National University School of Medicine, Busan, Korea
}

\begin{abstract}
Dear Editor:
Dermatofibroma is a common cutaneous nodule of unknown etiology that frequently occurs on the extremities. Although most dermatofibroma are usually asymptomatic, itching and pain are often noted in some patients. Furthermore, it is known to be the most common tumor among all painful skin tumors ${ }^{1}$. The pain of deep-seated dermatofibroma may be due to tumoral nerve invasion or mass effect on the nerve fibers ${ }^{2}$. However, there have been no studies investigating pain of typical dermatofibroma in the medical literature. Protein gene product 9.5 (PGP 9.5) is known as a sensitive neural/nerve sheath marker and preferably used for a wide range of cutaneous tumors. We quantitatively assessed the expression PGP 9.5-immunopositive nerve fiber bundles in patients with dermatofibroma with and without pain as previously published ${ }^{3}$.

This study was approved by the institutional review board of Pusan National University Hospital (IRB no.H-1812011-074), and the requirement for informed consent was waived. We retrospectively investigated the subjects diagnosed with dermatofibroma, all of whom underwent a
\end{abstract}

Received August 28, 2019, Revised November 27, 2019, Accepted for publication December 19, 2019

Corresponding author: Min-Young Yang, Department of Dermatology, Pusan National University Hospital, 179 Gudeok-ro, Seo-gu, Busan 49241, Korea. Tel: 82-51-240-7338, Fax: 82-51-245-9467, E-mail: yasmile@hanmail.net ORCID: https://orcid.org/0000-0001-8994-8401

This is an Open Access article distributed under the terms of the Creative Commons Attribution Non-Commercial License (http://creativecommons. org/licenses/by-nc/4.0) which permits unrestricted non-commercial use, distribution, and reproduction in any medium, provided the original work is properly cited.

Copyright (c) The Korean Dermatological Association and The Korean Society for Investigative Dermatology skin biopsy, and each specimen was stained immunohistochemically with an PGP 9.5 polyclonal antibody (1: 1,000; UltraClone, Cambridge, UK). Demographics and other clinical data were collected through our patient record database at PNUH from January 2013 to March 2019. We included patients with dermatofibroma that were smaller than $5.0 \mathrm{~cm}$ in diameter, located on leg, and histologically proven to be the common type. We subdivided patients into 2 groups according to the presence of pain or tenderness. The interpretation for intensity of PGP 9.5 expression were performed by experienced pathologist (one of the co-authors). In each specimen, the presence of nerve fiber bundles was comprehensively demonstrated with both hematoxylin-eosin and PGP 9.5 stains. Also, the number of PGP 9.5-immunopositive nerve fiber bundles was counted within the tumor mass and adjacent dermis aside from the main lesion, respectively, and compared quantitatively between the two compartments.

All statistical analyses were performed using IBM SPSS ver. 21 (IBM Corp., Armonk, NY, USA). For comparison of the investigated data between the groups, the chi-square test or Fisher's exact test were used for categorical variables, and the independent t-test was used for continuous variables. A $p$-value $<0.05$ was considered statistically significant.

Overall, 22 patients with dermatofibroma were included in the study (Table 1). Furthermore, 14 patients (63.6\%) complaining of pain or tenderness were assigned to group 1 ; and 8 patients $(36.4 \%)$ without pain or tenderness were assigned to group 2. No statistically significant differences were observed in the demographic characteristics between both groups, such as mean age or sex ratio. The average sizes of the lesions were $53.9 \pm 25.9 \mathrm{~mm}^{2}$ and 
$36.6 \pm 18.8 \mathrm{~mm}^{2}$ in groups 1 and 2, respectively, with mean durations of $15.1 \pm 10.6$ months and $17.8 \pm 12.3$ months in groups 1 and 2, respectively. There were no significant differences in lesion size or duration between patients with and without pain $(p>0.05)$.

In an analysis of the association between the immunopositive nerve fiber bundles and pain, nerve bundles were observed within the tumor and the adjacent dermis in 13 patients $(92.9 \%)$ and 12 patients $(85.7 \%)$, respectively, in group 1. However, in group 2, nerves were observed within the tumor of $6(75.0 \%)$ and the adjacent dermis of $5(62.5 \%)$. The number of immunopositive nerve fiber bundles within the tumor and adjacent dermis were variable (Fig. 1). Furthermore, 11 (78.6\%) and 2 (25.0\%) specimen in groups 1 and 2, respectively, showed more nerve fiber bundles within the tumor compared to the adjacent

Table 1. Clinical characteristics and distribution of nerve fiber bundles detected by protein gene product 9.5 (PGP 9.5) between different groups according to the presence of pain

\begin{tabular}{lccc}
\hline \multicolumn{1}{c}{ Variable } & Group 1* & Group 2 & $p$-value \\
\hline Age $(y r)$ & $31.4 \pm 6.7$ & $38.1 \pm 8.9$ & 0.057 \\
Sex & & & 1.000 \\
$\quad$ Male & $4(28.6)$ & $3(37.5)$ & \\
$\quad$ Female & $10(71.4)$ & $5(62.5)$ & \\
Size $\left(\mathrm{mm}^{2}\right)$ & $53.9 \pm 25.9$ & $36.6 \pm 18.8$ & 0.114 \\
Duration (mo) & $15.1 \pm 10.6$ & $17.8 \pm 12.3$ & 0.652 \\
Presence of nerve fiber bundles & & \\
$\quad$ Within tumor & $13(92.9)$ & $6(75.0)$ & 0.527 \\
$\quad$ Adjacent dermis & $12(85.7)$ & $5(62.5)$ & 0.309 \\
$\quad$ Within tumor $>$ & $11(78.6)$ & $2(25.0)$ & 0.026 \\
$\quad$ adjacent dermis & & & \\
\hline
\end{tabular}

Values are presented as number $(\%)$ or mean \pm standard deviation. *Patients with pain or tenderness $(n=14),{ }^{\dagger}$ Asymptomatic patients $(n=8) .{ }^{\ddagger}$ The number of cases that presented with more nerve fiber bundles within the tumor than the adjacent dermis. dermis. There were no significant differences in the proportion of cases that showed nerve fiber bundles between both groups $(92.9 \%$ vs. $75.0 \%, p=0.527 ; 85.7 \%$ vs. $62.5 \%, p=0.309)$. However, the predilection of PGP 9.5 expression within tumor mass were more pronounced in group 1 than in group 2, and this demonstrated a statistically significant difference $(78.6 \%$ vs. $25.0 \% ; p=0.026)$.

To our knowledge, this is the first comparative study to assess the pathogenesis of pain in dermatofibroma, especially with a PGP 9.5 stain. We found that dermatofibroma with pain had higher PGP 9.5-stained nerve fiber bundles within the tumor compared to the adjacent dermis. A previous study on benign dermal neoplasm demonstrated that dermatofibroma had only negative to weak staining for PGP 9.5, but these data only focused on a comparison with other tumors and did not correlate it to the clinical manifestation ${ }^{4}$.

In a study for the pathogenesis of pain in angioleiomyomas, a well-known painful tumor, most cases had nerve fibers immunoreactive for neural markers within the tumor stroma besides the capsule ${ }^{5}$. Likewise, although the mechanisms that lead to pain in dermatofibroma are not fully understood, the more abundant innervation within the tumor rather than the adjacent dermis may play a role in mediating pain.

We hypothesized that the mechanism of pain in patients with typical dermatofibroma was caused by nerves that are scattered within lesions and are likely to be mechanically compressed by densely proliferating fibrohistiocytic cells, which is similar to the pathological mechanism of pain in angioleiomyomas ${ }^{6}$.

However, our study has several limitations. First, the number of subjects were rather small for a statistical analysis of the pathological mechanism of pain in dermatofibroma. Second, although the margins of adjacent dermis were de-
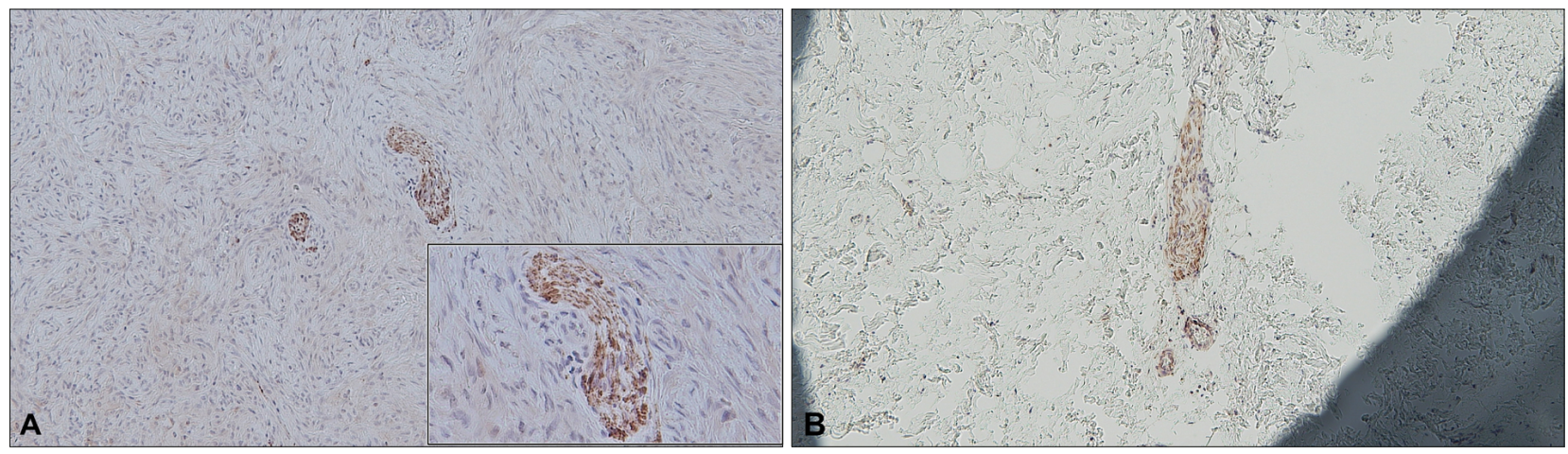

Fig. 1. Expression of protein gene product 9.5 (PGP 9.5) in representative specimens of dermatofibroma (within tumor mass and adjacent dermis, respectively, $100 \times$ ). (A) Granular pattern of immunostaining with PGP 9.5 highlighting nerve fiber bundles within the main mass (inlet image showing an enlarged view of the aggregates of nerve fibers, $200 \times$ ), and (B) those within the adjacent dermis. Black ink marking to distinguish areas away from the main lesion. 
fined as the area within a high-power field $(40 \times)$ from the outline of the tumor based on previous reports, a more strict and clear-cut definition of adjacent dermis is unclear because of a non-capsulated, poorly demarcated tumor.

Although our results must be interpreted in the context of the limitations, these provide information on the association between pain and its pathogenesis in dermatofibroma.

\section{CONFLICTS OF INTEREST}

The authors have nothing to disclose.

\section{FUNDING SOURCE}

This study was supported by the Biomedical Research Institute Grant (2018B024), Pusan National University Hospital.

\section{DATA SHARING STATEMENT}

The data that support the findings of this study are available from the corresponding author upon reasonable request.

\section{ORCID}

Kyung-Nam Bae, https://orcid.org/0000-0003-3254-8401

Hyun-Joo Lee, https://orcid.org/0000-0002-1088-0975

Sung-Min Park, https://orcid.org/0000-0002-4915-8111
So Jeong Lee, https://orcid.org/0000-0002-6465-9811

Moon-Bum Kim, https://orcid.org/0000-0003-4837-0214

Min-Young Yang, https://orcid.org/0000-0001-8994-8401

\section{REFERENCES}

1. Naversen DN, Trask DM, Watson FH, Burket JM. Painful tumors of the skin: "LEND AN EGG". J Am Acad Dermatol 1993;28(2 Pt 2):298-300.

2. Chung J, Namkoong S, Sim JH, Lee JS, Hong SP, Kim MH, et al. Deep penetrating benign fibrous histiocytoma of the foot associated with throbbing pain. Ann Dermatol 2011; 23(Suppl 2):S239-S242.

3. Giray B, Esim-Buyukbayrak E, Hallac-Keser S, KarageyimKarsidag AY, Turkgeldi A. Comparison of nerve fiber density between patients with uterine leiomyoma with and without pain: a prospective clinical study. Geburtshilfe Frauenheilkd 2018;78:407-411.

4. Wang GY, Nazarian RM, Zhao L, Hristov AC, Patel RM, Fullen DR, et al. Protein gene product 9.5 (PGP9.5) expression in benign cutaneous mesenchymal, histiocytic, and melanocytic lesions: comparison with cellular neurothekeoma. Pathology 2017;49:44-49.

5. Hasegawa T, Seki K, Yang P, Hirose T, Hizawa K. Mechanism of pain and cytoskeletal properties in angioleiomyomas: an immunohistochemical study. Pathol Int 1994;44:66-72.

6. Hachisuga T, Hashimoto H, Enjoji M. Angioleiomyoma. A clinicopathologic reappraisal of 562 cases. Cancer 1984;54: 126-130. 